\title{
VTS Bilingual Teaching Simulation Model Based on Constructivism
}

\author{
Weidong $\mathrm{Hu}$ \\ Department of navigation \\ wuhan university of technology \\ Wuhan, China \\ hwd998@163.com
}

\begin{abstract}
Simulation technology has becoming so popular that it has been increasingly applied in the fields of undergraduate education and vocational training. Internationally, it has also played a great role in training Vessel Traffic System (VTS) operators. Bilingual teaching of specialized course carried out with VTS simulator can greatly improve the study interest and enthusiasm of maritime management undergraduates who are lack of perceptual experience. The VTS simulation platform, which concords with the four key elements of constructivist learning environment, truly realizes the student-oriented philosophy. With the theoretical support of constructivism, the VTS simulation platform are constructed by utilizing simulation theories based upon object-oriented technologies and the bilingual teaching scheme with the support of simulation technologies is put forwarded.
\end{abstract}

Keywords- VTS course; constructivism; bilingual teaching mode

\section{INTRODUCTION}

Swiss Philosopher and Psychologist J.Piaget put forward the famous constructivism theory which is also called structuralism theory. The most important component of it is the constructivism learning concept, which emphasizes students' initiatives in exploring and discovering knowledge and constructing the significance of their studies [1].

VTS which means vessel traffic system is a professional course featured with strong application prospects opened by School of Navigation, Wuhan University of Technology for students major in the renewal Maritime Management. It is a extensive course which involves knowledge of radar, radar data processing, communication, computer and collision prevention and relevant international laws, regulations and standards issued by international organizations such as IMO, ITU and IALA. If we treat the students as machines that passively accept the knowledge by following traditional teaching schemes, undoubtedly they would become a flock of indigestive ducks.

In view of the fact that maritime management is internationally oriented, this research aims to design an interactive curriculum system based on the VTS stimulator platform by drawing upon the constructivism learning theory and explores the new bilingual teaching mode of professional courses supported by simulation technologies.

\section{TEACHING MODE OF CONSTRUCTIVISM}

The traditional teaching mode centers on teachers, who impart knowledge to students through explanation, blackboard writing as well as a variety of media and students passively accept what they are instructed. Thus, in this kind of teaching mode, teachers are the active instructors (imparters of knowledge) while students are passive recipients of external stimulations and targets of knowledge infusion.

The learning method advocated by constructivism learning concept is a student centered learning under the guidance of teachers. Constructivism learning environment contains four key elements, which are situation, cooperation, conversation and meaning construction. Therefore, we generalize the teaching mode that concords with the constructivism learning theory and constructivist learning environment as students centered, the teachers function as the organizers, instructors, assistors and promoters in the whole teaching process and utilize the learning environment elements such as situation, cooperation and conversation to enable students to fully play their initiative, enthusiasm and pioneering spirit, achieving the final goal of enabling students to effectively construct the significance of what they are studying.

\section{ESTABLISHMENT OF VTS ANALOG SiMULATION PLATFORM}

In VTS service, the key issue is the relationship between sea and land. It's significant to make accurate navigation for vessels from land. So, the coordination between VTS operators and marine navigators will affect greatly the sailing security and effects of the vessel.

\section{A. Construction Scheme of VTS Analog Simulation Platform}

A qualified VTS system should include fundamental functions as follows, data collecting, data process, data displaying and operate control, information transmission, communication and data saving and management. To fulfill the functional requirements mentioned above, VTS system needs to integrate data resource units (radar, radar signal recorder and radar signal simulator) radar data process equipment, analogical VHF communication unit (operation panel and communication sever),teacher work station, student VTS operating work station and students slipway 


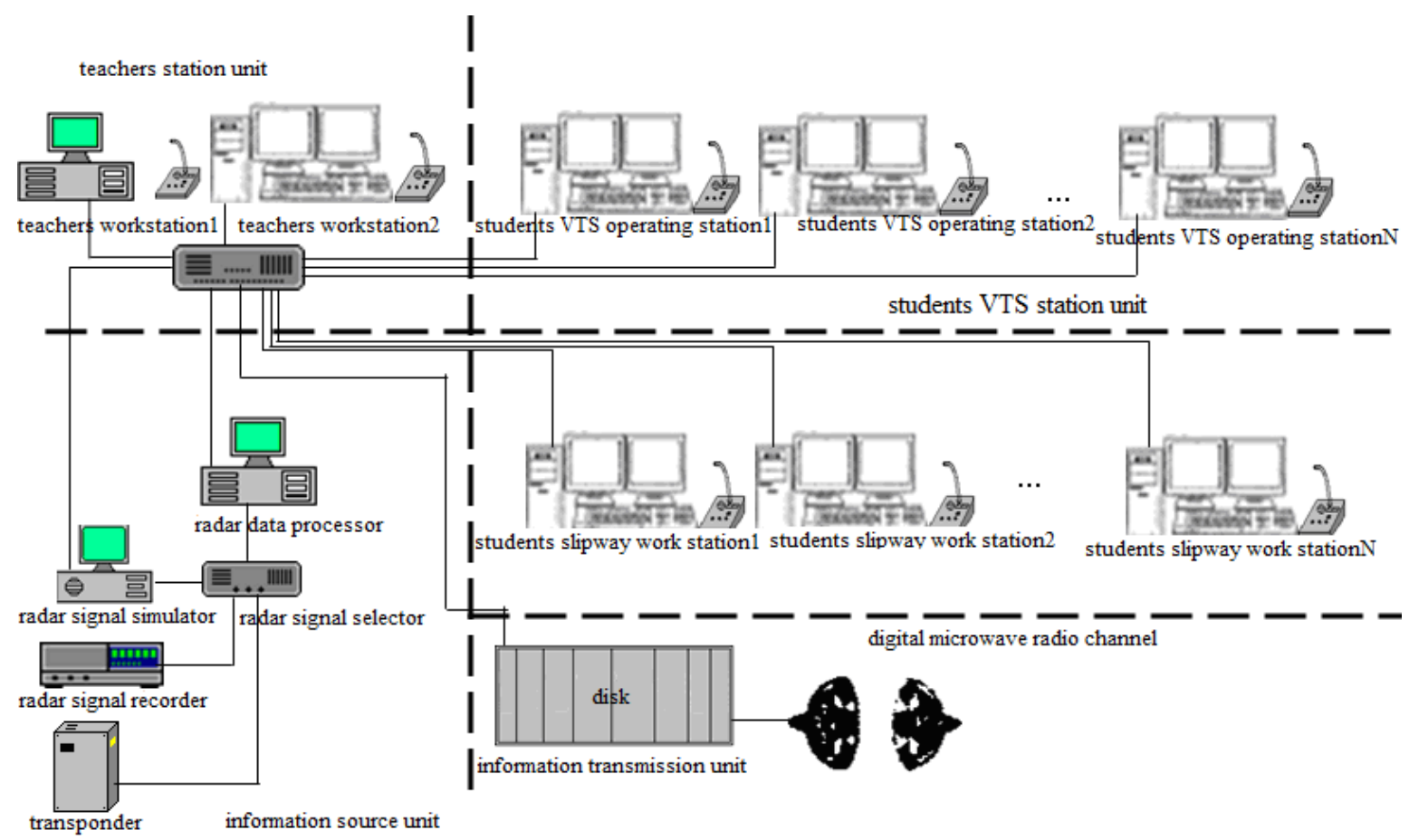

Figure1. Construction of VTS Analog Simulation System.

operating work station. System fundamental scheme is shown in Fig.1.

In Fig.1, each unit connects mutually via LAN. Transponder of data resource unit, radar signal recorder and radar signal simulator connect to radar data processor via radar signal selector ,the signal then be translated into data video signal and target tracing data and sent to teacher work station by radar data processor. Take practical consideration into account, two teacher work stations are set which is 1 and 2.Station 1 is to control radar signal simulator, setting radar targets parameters and motion trace, programming vessel code and used as VHF communication analog serve. Station 2 can be used to set target parameter and motion trace separately, producing data video and target data and send to VTS operational work station via LAN.

\section{$B$. The realization of the VTS system simulation technology}

The main part of the system is divided into modules such as ship target simulator, integrated information processing and display server, information processing and display client, remote platform simulator and APAR display simulator. Due to space limitations, here mainly introduces the design and implementation ideas and technology of the two key modules.

1) Ship target simulator: Ship target simulator mainly simulate ship data format by software, and send data to the client on the network in real time through TCP/IP protocol. Functions like presetting the route, adding ship target, and delivering regaining the authority of remote control are realized. a) Route adding: Mouse drawing on the chart, coordination of plotted route will be transformed, and then save the route data.

b) Ship adding: Set the starting point of the default route as provenance and the end of the route as the target destination. Speed and other attributes of the target are initialized. Ship speed can also be adjusted in the process of sailing at any time, the ship sailing is automatically according to the preset ship route, on the passage the control right can be transferred between local and remote.

2) Integrated information processing and display server. This module is mainly running a comprehensive information processing and display system, which is an integrated software platform use a vector chart as background, of which the function of chart zooming, roaming, selective amplification, graphic symbol mapping and hierarchical display operation can be achieved. The tracking target can be displayed in the chart background, combined with the function of multimedia, database, network and so on.

The implementation method of chart zoom in and out is the same, the only difference is the magnification coefficient is greater than 1 or less than 1 . To reduce visual sense of the flickering with the mouse in the process of chart zooming, the method of switching memory page is deployed in chart roaming. Selective amplification is fulfilled by technology of drawing in the dialog box. Plot of graphic symbols is in the top of the chart, of which the attribute information and geography data are stored in a special file. In hierarchical display, an additional property will be added to the belonged layer for each pixel, viz can be distinguished in hierarchical mapping [2] [3] [4]. 
a) Database: Take advantage of Microsoft Access, can store historical data for 30 days in a $6 \mathrm{~GB}$ hard disk, data before 30 days will be automatically deleted, operator should backup the important historical data each month. Interface with Access through $\mathrm{VC}++$, and database are updated along with the data source, hence all data is saved without missing [5].

b) Network function: The system includes a server and client. When the server dynamically processes and displays the target, any client linked in the network can also observe dynamically the same target in server. Historical data stored on server can also be looked back through the network via the playback function.

\section{BILINGUAL TEACHING MODE WITH THE SUPPORT OF SiMULATION TECHNOLOGY}

VTS course adopting bilingual teaching is because the service and management object of VTS is the ship from the world of different nationalities. The work languages specified by IALA and IMO are English, but the student's native language is Chinese. Bilingual class is the teaching mode of giving class in two languages which combine the traits of both general classroom teaching and English teaching and is with increased difficulty.

Bilingual teaching mode based on the support of simulation technology is divided into the following methods,

1) Multiple display modes: Create a variety of situations using simulation class and open up new ideas for the construction of learning environment for bilingual teaching, of which the more prominent ones are interested context, open situation and emotional context.

2) Bicultural comparison patterns: Simulation classroom can invoke live recording scene playback from English-speaking country VTS center to help students understand and feel the society and culture of the country effectively through the real picture and language.

3) Multi-role experience model: Multi-role experience is to allow students to attain the immersive reception and cognition from different roles. The simulation technology applied in bilingual teaching is to arouse the enthusiasm of the students learning.

4) Simulation playback evaluation model: Make full use of multimedia recording module in VTS analog simulation system. Multimedia recording module is developed on the basis of radar data recording equipment, which is usually designed as aggregation which includes multi-function recording and playback software module, and can record and replay all kinds of information running in VTS.

5) Multi-incentive example mode: According to the theory, in the bilingual class, example strategy can be fulfilled by using a VTS simulation platform to make students have a clear reference object and stimulating their internal motivation.

6) Multi-aspect learning mode: Multi-aspect practice refers to train students' English ability from the aspects of listening, speaking, reading and writing, thus promoting their comprehensive and coordinated development which is hardly achieve in the traditional classroom.

\section{CONCLUSION}

Start with the VTS function and the simulation modeling theory, key problems such as function description and modules of simulation system designed of VTS course are analyzed and discussed.

In the curriculum program planning of maritime management, simulation technology and the bilingual teaching theory are tentatively integrated by applying constructivism theory, and the unique bilingual teaching mode supported by simulation technology are designed.

\section{REFERENCES}

[1] Wang Xiurong, "Effective input for FLT: implications from constructivist learning theory," Journal of Hotan teachers college, Vol.26, Jul. 2006, pp.120.

[2] IMO model course 1.27. The operational use of electronic chart display and information systems (ECDIS) . 2000.

[3] Zhang yingjun, Math and Algorithmic Fundamentals of EDIS, 1st ed., Dalian: Dalian Maritime University Press, 2001.

[4] Zhu shili, Electronic Chart Application System Design, 1st ed., Beijing: National Defence Industry Press, 1997.

[5] Mr.Jurgen Meine, "Creation of a regional VTS with data fusion and integration of vessel data and movement details over the area of several independent ports, coastal and inland waterways," VTS 2004 Symposium. 